\title{
Subjectivation Éthique et Spiritualité Politique: À propos de L'Analyse de Michel Foucault sur la Révolution Iranienne
}

[Subjetivação Ética e Espiritualidade Política: Sobre a Análise de Michel Foucault acerca da Revolução Iraniana]

\section{Nelson Fernando Roberto Alba}

Résumé: L'article propose une restitution des aspects principaux de la lecture foucaldienne sur la Révolution iranienne à partir de l'analyse «des reportages d'idées» que le philosophe fait en Iran en tant que correspondant entre 1978 y 1979 . Dans ce sens, le texte fait une mise en contexte du panorama politique iranien avant de la soi-disant «Révolution», lequel peut être entendu sous l'optique d'un diagramme stratégique de relations de pouvoir. Cela dit, on attire l'attention sur les notions de "volonté collective» et la «subjectivation éthique» produites pendant le soulèvement iranien, ainsi que la notion de "spiritualité politique» comprise comme une pierre de touche pour l'agencement d'une expérience politique collective hétérogène à l'égard du pouvoir politique stratégique.

Mots-clés: Subjectivation collective, éthique, spiritualité politique, volonté collective.

Resumo: O artigo propõe apresentar os principais aspectos da leitura foucaultiana da "Revolução iraniana" a partir da análise da "reportagem de ideias" que o filósofo faz no Irã como correspondente entre 1978 e 1979. Nesse sentido, o texto contextualiza o panorama político iraniano antes da chamada "Revolução", que pode ser entendido a partir da perspectiva de um diagrama estratégico de relações de poder. Chama-se imediatamente a atenção para as noções de "vontade coletiva" e "subjetivação ética" surgidas durante a insurreição iraniana, bem como a noção de "espiritualidade política" entendida como uma pedra de toque para a montagem de uma experiência política heterogênea ao poder político estratégico.

Palavras-chave: Subjetivação coletiva, ética, espiritualidade política, vontade coletiva.

\footnotetext{
${ }^{*}$ Professeur à la Faculté de Philosophie et Lettres, Université Santo Tomás, Bogotá (Colombie). Doctorant en philosophie au Laboratoire Logiques Contemporaines de la Philosophie (LLCP) de l'École Doctorale Pratiques et Théories du Sens (ED 31) de l’Université Paris 8-Vincennes-Saint Denis. Contact: nelsonalba@hotmail.com. ORCID: https://orcid.org/0000-0003-2806-7162.

Je remercie au professeur Frédéric Rambeau du Département de Philosophie de l'Université Paris 8-Vincennes Saint-Denis, par son soutient académique pendant la rédaction du mémoire de Master Philosophie dont cet article est un produit partiel. Pour une analyse plus approfondie des implications politiques du schéma du «souci de soi» dans le contexte de la lecture foucaldienne de la Révolution iranienne voir RAMBEAU, F. "La política espiritual” [«La politique spirituelle»], Ciencia Política, Bogotá, v.10, n.19, p. 197-192, janvier-juin 2018.
} 


\section{Introduction}

La critique de certains auteurs $\mathrm{du}$ " poststructuralisme » qui, se réclamant des philosophes du soupçon (Nietzsche, Marx, Freud), conçoivent la religion en tant qu'assujettissement et intériorisation de la loi, est bien connue. C'est le cas, chez Michel Foucault, de l'analyse de la tradition judéochrétienne comme gouvernement des âmes dans la notion de " pouvoir pastoral ». Cependant, la lecture que le philosophe a pu avoir de la religion dans sa vaste œuvre ne se restreint pas à une simple critique envers le christianisme. En effet, ses réflexions sur le propos sont variées et hétérogènes, comme l'atteste son analyse de la Révolution iranienne entre 1978 et 1979 .

En effet, en tant que correspondant du journal italien Corriere della sera, Foucault voyage en Iran en 1978 et écrit une série de reportages sur la Révolution iranienne qui ont suscité une grande polémique en France. En Iran le philosophe assiste à une mise en oeuvre de la politique incompatible avec le schéma classique de la modernité européenne. Il s'agit d'une " grève politique généralisée ", d'un appel populaire échappant à la rationalisation stratégique de la politique dans la mesure où elle est produite par une subjec- tivation massive enracinée dans une pratique spirituelle, celle de l'islam chiite. La religion est comprise ici non seulement comme l'obéissance externe à une loi, mais aussi comme une "vie spirituelle profonde », une " expérience spirituelle " grâce à laquelle les iraniens cherchaient un changement radical dans leur subjectivité.

Le philosophe voit dans les événements en Iran une scène irréductible aux catégories d'analyse occidentales, qui met par-là radicalement en cause le diagramme stratégique de l'époque, ce qui lui permet de penser autrement l'éthique et la politique même à l'égard de son propre travail analytique. En Iran Foucault assiste à l'effectuation d'une subjectivation éthique collective, d'un rapport à soi non individualisé et tellement disparate à l'égard du schéma de la gouvernementalité et de la maîtrise de soi qui en découle, que le philosophe n'hésite pas à affirmer que cette subjectivation collective constituerait en même temps une politique qui ne serait plus un gouvernement des autres. En somme, il s'agirait non seulement d'une esquive aux techniques d'individualisation et aux procédures totalisatrices façonnées par l'État en tant que rationalité politique, mais encore d'une esquive au gouvernement 
de la vie des individus et de leur gestion en tant que population.

Ainsi, à titre d'hypothèse on pourrait remarquer que la subjectivation collective produite en Iran a pu constituer une dimension extérieure, un certain dehors aux relations de pouvoir et aux stratifications du savoir qui composent le diagramme de la gouvernamentalité. Or mettre à l'épreuve cette hypothèse implique une difficulté majeure qui risque même de faire basculer l'analyse faite du philosophe sur la singularité de l'événement: la subjectivation collective qui, en Iran, a fait tomber le régime du shah, ne peut être comprise qu'à partir d'une expérience spirituelle qui renvoie directement à une conscience religieuse enracinée dans l'orthodoxie millénaire de l'islam chiite. Comment rendre compatible l'appel à un changement radical de la subjectivité avec la revendication d'une identité préconçue et fixe? Comment cette nouvelle subjectivité enracinée dans la spiritualité religieuse pourrait-elle constituer une politique autre que celle du pouvoir politique et stratégique iranien? N'était-ce pas Foucault lui-même qui posait la désindividualisation, la désidentification comme une sorte de condition pour se subjectiver et devenir autre? À la fin de sa vie, la lecture critique que le philosophe faisait des techniques d'assujettissement en Occident, dans le cas du christianisme par exemple, n'était-elle pas conçue comme une sorte d'intériorisation de la loi?

Toutes ces indications nous suggèrent qu'il s'agit ici d'une lecture anti-stratégique, c'est-à-dire d'une prise de position faite par Foucault à propos de la "révolution iranienne », mais qui reste hétérogène à l'égard de ses propres analyses théoriques. En ce sens, et grâce au caractère paradoxal qui entraîne l'analyse du philosophe sur ces événements, cet article poursuit un double objectif. D'abord, établir l'idée d'une éthique incompatible avec le schéma de la maîtrise de soi et par conséquent, incompatible aussi avec l'idée de la liberté individuelle sous-jacente au diagramme de la gouvernementalité. Ensuite, explorer la face politique que peut prendre cette subjectivation éthique collective vis-à-vis du pouvoir politique traditionnel en Iran.

On commencera par faire une brève mise en contexte du panorama politique iranien, en essayant de remarquer l'existence d'un diagramme stratégique des relations de pouvoir en Iran face auquel se produit une éthique et une politique antistratégiques. Après, on examinera le possible rapport entre l'idée de "volonté 
collective " décrite par le philosophe et la subjectivation éthique non individuelle produite en Iran à l'égard de la singularité du chiisme comme expérience spirituelle qui est à la base des événements. Ensuite, on analysera la notion de "spiritualité politique », conçue par Foucault comme un " principe de création politique » en la rapportant au "soulèvement » comme figure d'une nouvelle politique subsidiaire de la subjectivation éthique collective iranienne. Finalement, on essayera de dégager des problématiques impliquées dans l'analyse par rapport à une éthique et une politique non-stratégiques et aussi on fera quelques remarques à propos du statut de l'analyse de la révolution iranienne chez le philosophe.

\section{Révolution iranienne: politi- que et éthique stratégique}

Si le stratège est un homme qui dit: "Qu'emporte telle mort, tel cri, tel soulèvement par rapport à la grande nécessité de l'ensemble et que m'importe en revanche tel principe général dans la situation particulière où nous sommes »[...] ma morale théorique est in- verse. Elle est « antistratégique »: être respectueux quand une singularité se soulève, intransigeant dès que le pouvoir enfreint l'universel. Choix simple, ouvrage malaisé: car il faut tout à la fois guetter, un peu au-dessous de l'histoire, ce qui la rompt et l'agite, et veiller un peu en arrière de la politique sur ce qui doit inconditionnellement la limiter. (FOUCAULT, 2001n, p. 794).

Malgré son caractère crypté, l'affirmation est essentielle dans la mesure où elle nous permet d'entrevoir une sorte de dimension incompatible avec le travail analytique de Foucault, à savoir l'antistratégie. Et avant tout: qu'est-ce que la "morale théorique " du philosophe? On aurait tort d'en donner une définition, mais au moins pour nous il s'agit d'une impulsion critique et entièrement empirique qui donne naissance aux ouvrages de Foucault et en même temps échappe à leurs implications théoriques. Quel statut peut-on donner à ces affirmations lorsque c'est Foucault lui-même qui conçoit la stratégie comme principe d'intelligibilité du pouvoir politique et qui, d'ailleurs, considère la 
résistance comme étant aussi instable, inventive et stratégique que les relations de pouvoir? En effet, la date, 1979 et le contexte dans lequel s'énonce cette affirmation - la révolution iranienne - sont très significatives pour qui veut prendre en compte les considérations du philosophe sur sa morale théorique antistratégique. Face à une politique et une éthique - ou plutôt une morale - de son époque qui agit à partir des rationalités pratiques et des calculs normés, le philosophe opposerait, non plus un renversement tactique opéré à l'intérieur d'un diagramme de pouvoir, mais son propre engagement théorique, sa façon d'agir en tant qu'intellectuel «spécifique».

Daniel Bensaïd rappelle précisément les "grandeurs » et les " misères » de Foucault à l'égard de la crise de la politique et sa compréhension stratégique à cette époque. Grandeur, car c'est Foucault parmi d'autres qui annonce l'effondrement du paradigme politique de la modernité, lequel se reflétait dans un obscurcissement des horizons stratégiques », c'està-dire dans une crise de la politique elle-même: " c'est bien la stratégie qui est en cause. Car une politique sans stratégie ne peut être rien d'autre qu'une gestion apeurée d'un quotidien, qui se répète et piaffe sur place »(Bensaïd, 2009, p.3). Après d'avoir as- sisté à une séquence d'une "forte intensité stratégique " où le problème du pouvoir était posé dans l'urgence des partages du monde, du tracé des territoires, des affrontements systématiques, des révolutions et, à la limite, des émancipations, le travail de Foucault aurait aperçu et traduit une crise stratégique naissante qui ne supposait rien d'autre qu'une mise en cause de la politique telle que l'Occident l'a comprise depuis Machiavel, Rousseau jusqu'à Marx et Lenin (peuple, classe, souveraineté, territoire, nation, etc.). D'ailleurs, pointe Bensaïd, ce travail critique sur la crise des stratégies d'émancipation s'est vu accompagné aussi par l'annonce d'une crise de la raison historique et une crise des théories critiques à l'égard de la même modernité. Misère puisque Foucault revendique une lecture stratégique pour analyser le pouvoir paradoxalement « au moment où s'effacent les paramètres d'une pensée stratégique ». Bensaïd parle des années quatre-vingt comme le moment d'un " degré zéro de la stratégie », comme le résultat d'un désenchantement du politique conduisant à la conclusion qu'il n'y plus de solution gagnante possible et il n'y a plus de place pour aucune stratégie: "lorsqu'elle atteint son degré zéro, il ne reste plus qu'un impératif catégorique 
moral de résistance et un formalisme de la fidélité. L'éthique de la politique s'évanouit alors dans le moralisme antipolitique ». En somme: « l'abdication stratégique va logiquement de pair avec le renoncement à une théorie qui ne soit, depuis Clausewitz, ni une science ni un art (au sens de simple savoir-faire empirique), mais un concept stratégique des forces et des antagonismes en mouvement » (Bensaïd, 2009, p.3).

Évidemment, il s'agit ici d'un questionnement fait sur Foucault à partir de la critique que ce dernier formule du marxisme et, dans ce sens, ce n'est pas que le philosophe n'ait pas vu dans le marxisme une analyse stratégique de la lutte de classes, mais plutôt que Foucault attire l'attention sur le besoin de penser l'antagonisme concernant le modèle de la lutte de classes à partir d'une méthode stratégique. Et ainsi, tant le propos de Bensaïd que celui de Foucault semblent être bien divergents. Cependant, la lecture de Bensaïd nous permet de remarquer que la discussion du philosophe à propos de la stratégie comme principe d'intelligibilité du pouvoir politique passe par le marxisme, mais ne s'y réduit pas. Quant au diagramme des rapports de force qui constitue tout le corps social, Foucault y inscrit non seulement l'analyse philosophique et sociologique du hégélo-marxisme, mais aussi une conception de la politique autre qu'il nomme Realpolitik et qui désignerait une politique étrangère fondée sur le calcul des forces et l'intérêt national, autrement dit une politique internationale et pragmatique conçue à partir des intérêts politiques et économiques des grandes puissances. En somme, une politique stratégique grâce à laquelle les états contemporains interagissent au profit de leurs intérêts particuliers en excluant toute sorte de principes moraux.

Ainsi, on peut remarquer que c'est face à cette idée de la politique comme la Realpolitik que Foucault a formulé, non seulement ses analyses sur le pouvoir politique comme un calcul stratégique, mais aussi ses propres affirmations sur sa morale antistratégique. Dans ce contexte, pour nous, la lecture foucaldienne de la soi-disant "Révolution iranienne " se constitue dans un évènement qui permet de faire une nouvelle analyse et une nouvelle sémantique de la politique, du temps et de l'histoire. Bien sûr, on n'a pas l'intention de faire une reconstruction des événements et encore moins de juger les considérations du philosophe sur ce propos. Pour nous il s'agit plutôt d'aller au cœur des analyses sur deux notions qui se trouvent dans 
ce contexte, à savoir la «volonté collective » et la «spiritualité politique ». Mais avant d'en dégager des éléments pour notre recherche, il faudrait préciser le caractère stratégique de ces événements tel que le conçoit Foucault.

\section{La modernisation: un régime ar- chaïque}

Foucault se rend à deux reprises en Iran: entre le 16 et le 24 septembre et entre le 9 et le 15 novembre 1978. C'est dans cette période que le philosophe, non seulement écrit une série de "reportages » pour le journal italien Corriere della sera, publiés aussi en France et provoquant une forte polémique dans l'opinion publique, mais aussi propose de constituer une équipe d'intellectuels reporteurs chargés: " [d'] assister à la naissance des idées et à l'explosion de leur force: et cela non pas dans les livres qui les énoncent, mais dans les événements dans lesquels elles manifestent leur force » (FOUCAULT, 2001h, p.707). Ce cadre dans lequel s'inscrit l'analyse de Foucault à propos des événements en Iran nous permet d'entrevoir son entreprise intellectuelle: non pas celle de restituer les causes et d'explorer les conséquences de la révolution iranienne selon une approche théorique d'un académicien, encore moins celle d'un journaliste de bureau voulant tout saisir des événements, mais celle de l'intellectuel qui sans la prétention de tout juger assiste aux événements et essaie de rendre intelligible leurs foyers de problématisation.

Il faudra d'abord distinguer "l'âme du soulèvement » et sa manifestation réelle au travers ce qui s'est passé en Iran entre 1978 et 1979. La distinction est essentielle dans la mesure où elle replace notre analyse dans la perspective des considérations de Foucault à propos de l'âme des événements en Iran, c'est-à-dire de quelque chose qui faisait partie des événements, mais qui en même temps échappait à leur effectuation. Certes, s'agissant de la révolution iranienne et de la lecture qu'en fait le philosophe, on pourrait évaluer les considérations de Foucault, et même remarquer à quel point le philosophe s'est trompé dans sa lecture passionnée sur le rôle initial de l'Ayatollah Khomeiny dans ce soulèvement populaire. Mais, ce faisant, on constituerait un tribunal qui critiquerait ou exempterait le philosophe de ses fautes et de ses points d'aveuglement dans l'analyse d'un événement qui lui était étranger.

Ainsi, on est loin d'affirmer que la lecture de la révolu- 
tion iranienne ne constituerait que le passage ou un point intermédiaire entre l'analyse des formes d'assujettissement propres du modèle stratégique et l'analyse des pratiques de subjectivation dans l'antiquité grécoromaine. Ce qui supposerait une certaine adéquation de cette lecture de Foucault à une logique d'ensemble considérée comme le fil rouge de son œuvre. De telle sorte, et malgré les difficultés et les paradoxes qu'entraîne l'analyse du philosophe, notre objectif est d'affirmer l'hétérogénéité de son analyse à l'égard de la logique de la gouvernementalité et des idées de politique et d'éthique qu'elle comporte. Et, à la limite, d'y voir une effectuation antistratégique.

En Iran l'existence d'un État impérial autour d'une dynastie royale, celle des Pahlavi mise et maintenue grâce à l'intervention constante des anglais et des américains pendant le XXe siècle, nous suggère qu'il s'agit ici d'une politique stratégique, c'est-à-dire de l'exercice d'une forme de rationalisation ne concevant l'action politique qu'à l'intérieur d'un calcul opératif, lequel ne correspond pas la plus part du temps ni aux intérêts des gouvernés ni encore moins aux conditions matérielles réelles dans lesquelles cet exercice devrait se donner. En effet, depuis 1921 il y a en Iran une dynastie instaurée suite au coup d'État de Reza Khan, avec l'appui des anglais et qui avait fondé les premiers pas d'un processus de modernisation important: fondation des universités, construction de chemins de fer et une industrialisation massive qui finit par faire basculer l'ordre social du pays. En 1941 Reza Khan est forcé d'abdiquer par l'invasion des britanniques et des soviétiques, mais son projet de modernisation du pays est poursuivi par un gouvernement démocratique, celui de Mohammad Mossadegh - notamment caractérisé par la nationalisation du pétrole, la création de la sécurité sociale et la mise en œuvre d'une réforme agraire -. Il est à son tour renversé par un coup d'État orchestré à l'aide des américains en 1953 et qui a replacé le fils de Reza Khan, Mohammad Reza comme shah d'Iran jusqu'à sa chute en 1979 par la révolution iranienne.

D'après Foucault « la modernisation comme projet politique et comme principe de transformation sociale est en Iran une chose du passé " (FOUCAULT, 2001c, p.680). Pourquoi? D'abord parce que les tentatives de moderniser l'Iran à " l'européenne » datent des années vingt et se sont nourries d'un modèle de modernisation implémenté par Mustafa Ke- 
mal en Turquie sur la basse de trois principes: nationalisme, laïcité et modernisation. Ensuite, parce que cette soi-disant modernisation telle qu'elle a été conçue par le shah relève d'un archaïsme : elle comporte la revendication d'une aryanité, d'un mythe fondé sur l'idée d'une race aryenne pure appartenant à une civilisation Perse préislamique dont les iraniens doivent se réclamer - le nom Iran signifie " royaume des Aryens » et il a été décrété par le shah en 1935-. En somme, le projet de modernisation de l'Iran passe impérativement par la tentative de diminuer le rôle de l'islam chiite en tant que forme de vie et vrai principe de conscience nationale. C'est pourquoi le philosophe affirme:

J'ai eu alors le sentiment de comprendre que les événements récents ne signifiaient pas le recul des groupes les plus retardataires devant une modernisation trop brutale ; mais le rejet, par toute une culture et tout un peuple, d'une modernisation qui est en elle-même un archaïsme. (FOUCAULT, 2001c, p.680).

D'ailleurs, cette imposition d'un régime archaïque par une dynastie royale se voit forcément accompagnée de l'usage et du maintien des forces armées. Mais ici, curieusement, d'après le philosophe, "il n'existe pas en Iran de structure économico-militaire solide » (FOUCAULT, 2001b, p.666) puisque, en l'absence d'une armée avec une force d'intervention politique, il n'y pas une earmée, mais quatre: un armée traditionnelle chargée de surveiller et administrer le territoire, un service de sécurité intérieure et de renseignement secret fortement critiqué par ses actions violentes - la SAVAK -, une armée de combat avec des armements sophistiqués et une autre armée composée par 30000 conseillers américains.

Faute stratégique du shah dès ses premières tentatives de modernisation d'Iran, l'usage excessif de la force s'est constitué comme une pratique systématique pour apaiser la croissante contestation sociale. Dans ce sens, le massacre du "vendredi noir ", dans lequel l'armée tire sur une foule massée dans la place Djalehen à Téhéran provocant entre 3000 et 4000 mille morts, est considérée comme une des actions les plus sanglantes perpétrées par le régime du shah. De plus, c'est dans les multiples manifestations religieuses qu'on peut repérer le début d'un soulèvement 
populaire: la commémoration des victimes de cet événement ainsi que d'autres comme la mort de 377 assistants à la projection d'un film sur la contestation paysanne à cause d'un incendie à la ville d'Abadan.

Mais s'il est certain que cette dynastie royale a réveillé en Iran un malaise social généralisé en s'emparant du pouvoir politique par la force et en imposant un archaïsme comme régime de modernisation, il n'en reste moins vrai que ce malaise généralisé s'est rendu d'abord manifeste à cause des conditions économiques déplorables partout dans le pays. En effet, la mise en œuvre d'un système de développement depuis 1950 a bouleversé l'économie traditionnelle du pays pendant 25 ans: d'un pays où l'agriculture féodale et l'élevage sont les activités économiques les plus répandues dans la population et l'origine d'une concentration démographique majeure dans les zones désertes, on passe à un pays devenant l'un des producteurs majeurs de pétrole de la sphère occidentale ; cela a forcément entraîné une augmentation considérable des revenus, mis au service d'une industrialisation massive et d'une intégration accélérée de l'Iran au marché international. D'où non seulement l'émergence d'une nouvelle cou- che sociale riche, laïque et bourgeoise due à une explosion démographique dans les centres urbains, mais aussi au détriment d'une couche sociale obligée à s'installer dans les quartiers pauvres de ces mêmes villes.

À ce propos Foucault remarque l'échec des grandes entreprises du pouvoir en Iran depuis 1963 et leur rejet par toutes les classes sociales. Mécontentement des grands propriétaires et des petits paysans à cause de la réforme agraire: les uns parce qu'ils étaient obligés de céder partie de leurs terrains, les autres, car ils devaient s'endetter et émigrer en ville. Mécontentement des artisans et des petits industriels à cause de l'arrivée continuelle d'exportations au détriment de leur économie. Mécontentement des commerçants de bazar lorsqu'ils ne trouvaient plus de petits villages à cause de l'urbanisation. Mécontentement, en dernière instance, de la classe riche attendant toujours un développement national industriel qui n'arrive pas. Or, affirmer cet aspect ne suppose pas de réduire la contestation populaire à une lutte économique, c'est-à-dire à la revendication d'un changement des conditions économiques dans lesquelles les iraniens étaient soumis depuis 30 ans. Évidemment, l'émergence du soulèvement ira- 
nien passe par une mise en question des conditions économiques, mais elle ne s'y réduit pas. Et, en ce sens, l'entreprise de Foucault consiste à montrer comment, en Iran, il n'y pas de lutte contre la domination politique, et encore moins contre l'exploitation économique, mais bien contre des formes d'assujettissement. C'est-àdire une lutte pour la constitution d'une nouvelle forme de subjectivité arrivant à faire tomber le régime du shah grâce à l'effectuation de la «volonté collective » de tout un peuple, laquelle se constitue sur la base d'une conscience religieuse qui fonde une spiritualité politique. Mais qu'est-ce que cette volonté collective et comment le philosophe la conçoit? Pourquoi peut-on parler de "subjectivation collective » et comment se rapporte-t-elle à une spiritualité dite religieuse?

\section{II - Éthique et spiritualité: de la volonté à la subjectivation col- lective}

Dans la préface de l'édition américaine de L'Anti-CEdipe publiée en 1977, Foucault conçoit le travail de Deleuze et Guattari comme étant «le premier livre d'éthique que l'on ait écrit en France depuis assez longtemps » (FOUCAULT, 2001a, p.134). Ici l'indication à propos de l'éthique renvoie d'emblée à " un style de vie, un mode de pensée et de vie " au point que le philosophe considère le livre comme un "art de vivre", une introduction à la vie non fasciste, un "guide de la vie quotidienne » qui se voit accompagné de principes essentiels pour se débarrasser d'un fascisme "qui hante nos esprits et nos conduites quotidiennes ».

Pour nous cette référence, même si elle semble être mineure, devient fondamentale pour comprendre l'idée que Foucault a pu avoir de l'éthique à cette époque. Lorsqu'il s'agit de l'interprétation foucaldienne des événements d'Iran, notamment à propos de l'éthique comme subjectivation collective, on aurait tort d'imposer à cette lecture une définition postérieure de l'éthique, celle de L'usage des plaisirs ou Le souci de soi (1984), comme singularisation de la conduite en tant que rapport immanent de soi à soi. En revanche, en Iran le philosophe assiste à l'effectuation d'une subjectivation collective, à l'émergence d'un nouveau type de subjectivité qui échappe au gouvernement par l'individualisation et la totalisation stratégique de la politique, ainsi qu'à la codification, systématisation et à la normalisation juridique de la conduite morale 
des sujets imposés par le régime du shah. C'est juste face à ce type de jeu stratégique de la politique et de l'éthique iranienne que Foucault oppose les catégories de "volonté collective » et de "gouvernement islamique ».

Volonté générale et Volonté collective: la grève de la politique

«L'Iran est actuellement en état de grève politique généralisée. Je veux dire en état de grève par rapport à la politique. » (FOUCAULT, 2001f, p.702). D’après Foucault, le soulèvement populaire qui s'est produit en Iran ne peut pas être conçu comme une contestation politique: si celle-ci rapporte à l'idée du calcul et de la stratégie traditionnelle de la politique, alors la "volonté politique » du peuple iranien "c'est de ne pas donner prise à la politique ». Bien avant d'être considérée comme politique - au sens de l'expression d'une résolution univoque et vide de tout un peuple - la volonté des iraniens est anti-politique, elle rejette un prolongement du régime du shah et de ses appareils de gouvernement et, d'ailleurs, elle refuse de faire immédiatement la place à une "bataille politique " sur le futur de l'Iran. Certes, le philosophe parle de volonté politique pour remarquer que ce soulèvement n'a pas de politique et en même temps afin de montrer toute sa puissance politique ; bien sûr, il s'y agirait d'une politique autre "parce que la politique n'est pas ce qu'elle prétend - l'expression d'une volonté collective ; elle ne respire bien que là où cette volonté est multiple, hésitante, confuse et obscure à elle-même " (FOUCAULT, 2001f, p.702). Ainsi, en définissant le soulèvement comme une grève par rapport à la politique, Foucault non seulement le détache d'une conception classique de la philosophie politique, mais aussi l'inscrit dans le domaine d'une politique spirituelle sur laquelle on reviendra par la suite.

Certes, Foucault parle de "volonté collective " par rapport à l'expression d'une volonté politique des iraniens qui est elle-même une grève de la politique. Cependant il ne faut pas croire que cette volonté collective serait une création ex nihilo des iraniens; il s'agit plutôt de l'apparition extraordinaire d'une catégorie très discutée, mais quelquefois mise en œuvre, à savoir la "volonté générale ». En effet, d'après Foucault cette « volonté générale » ne serait qu'un mythe censé être utilisé par les juristes et les philosophes pour analyser et justifier n'importe quel type d'institutions. Il s'agirait d'un " instrument théorique ", 
de quelque chose d'abstrait qui exprimerait l'accord des intérêts particulières pour tendre vers le bien commun de toute une société, mais que pour Foucault a toujours été une généralité introuvable. En revanche, en Iran cette "volonté générale » propre du contractualisme moderne et contemporain s'est donné un objectif précis et, en tant que telle, elle ne pourrait plus être considérée comme générale, mais comme " collective »: " cette volonté collective, qui dans nos théories, est toujours générale, s'est fixé, en Iran, un objectif absolument clair et déterminé, ainsi a-t-elle fait irruption dans l'histoire. » (FOUCAULT, 2001j, p.746).

D'autre part, on peut signaler la façon dont le philosophe distingue sa lecture des événements d'Iran de ses propres analyses théoriques. En Iran le soulèvement n'est pas le résultat d'un point de décrochage entre le pouvoir politique du shah et la subjectivation éthique du peuple, autrement dit, l'éthique comme transformation des modes de penser et de vivre des iraniens n'émerge pas comme un effet dérivé du pouvoir politique. Il ne s'agit aucunement d'un " renversement tactique »opéré par les iraniens à l'intérieur du diagramme de pouvoir politique, dans la mesure où le pouvoir politique en
Iran était une dynastie royale qui voulait imposer une forme de vie modernisée, libérale et en même temps attachée à un discours archaique revendiquant une race aryenne préislamique. En Iran il y a plutôt une subjectivation éthique collective comportant la revendication d'une spiritualité religieuse qui, à un moment donné, a joué un rôle antagoniste face au régime du shah et qui, pourtant, peut être considérée comme étant une politique autre, une spiritualité politique. Inversion du schéma politique-éthique par un lien éthique-politique, et basculement du modèle stratégique propre de l'analytique du pouvoir, telles semblent être les enjeux de la lecture que Foucault fait du soulèvement iranien.

En quoi consiste cette "volonté collective »dont parle le philosophe à propos des événements d'Iran et qu'il conçoit comme étant la manifestation de la volonté multiple de tout un peuple? D'abord elle a comme caractère essentiel le multiple et le disparate, le fait d'hésiter sur ellemême. Tel est le cas, par exemple, du panorama politique et social en Iran: du côté des mouvements politiques d'opposition on peut signaler Le Front National composé par des intellectuels libéraux urbains revendiquant un discours nationaliste proche de 
la social-démocratie, l'Union des Combattants Communistes se réclamant des thèses du marxismeléninisme et souvent associée à l'extrême gauche urbaine, et le Parti Communiste iranien (Toudeh) regroupant une partie importante de la population ouvrière du pays. Ces mouvements politiques avaient certes une forte orientation laïque et démocratique, mais celle-ci était la seule opposition au régime du shah? Certainement pas.

En ce qui concerne le clergé chiite, religion majoritaire en Iran, son opinion sur le départ du Shah est loin d'être unitaire. Car bien qu'en général le clergé soit d'accord avec la fin de la monarchie archaïque du Shah, la façon dont cette fin devait se faire et l'objectif qu'elle visait étaient un sujet de désaccord parmi les religieux. Ainsi, la posture de l'ayatollah Shariatmadari, la plus haute autorité religieuse en Iran à cette époque, établissait que le chiisme ne pouvait pas revendiquer un pouvoir temporel et devait laisser la place à un gouvernement de type républicain proche des intérêts du courant laïque des défenseurs des droits de l'homme. Cependant, cette vision plus spirituelle du chiisme entrait en conflit avec celle de l'ayatollah Khomeyni, une autre grande autorité religieuse opposée eu régime du shah qui vivait exilé à l'étranger depuis 1964. En effet, d'après le discours de plus en plus radicalisé de Khomeyni, non seulement la monarchie du shah est un système inadéquat pour l'Iran, mais aussi n'importe quel type de gouvernement en tant qu'il n'est pas détenu par une autorité religieuse. S'il peut donc bien y avoir une politique menée par les hommes, une démocratie, celle-ci doit être subordonnée au pouvoir absolu des religieux, le conseil des "Gardiens de la Révolution », et notamment d'un "guide suprême ». Il s'agissait en somme d'une politique menée par les hommes, mais entièrement fondée et conçue sur les principes de l'islam, c'est-àdire d'une "République islamique ».

Ainsi, concernant l'opposition au régime du shah, on aurait tort de proposer une lecture univoque tout à fait adéquate avec l'idée vide d'une volonté collective. Or ce que Foucault volonté collective correspond plutôt à la dizaine de groupes révolutionnaires, mouvements syndicaux, partis politiques, groupes religieux, tous ayant leur propre vision de l'avenir de l'Iran sans se soucier qu'ils soient des factions libérales, marxistes, anarchistes, laïques ou religieuses. De manière que pour nous cette volonté collective se présente tout d'abord comme 
étant entièrement disparate, hésitante et confuse, dans la mesure où elle exprime le sentiment d'une population qui jamais n'a été une unité.

A cet égard, il y a trois causes de l'accélération du soulèvement rapportées dans la notion de volonté collective qui attirent l'attention de Foucault: a) la population s'oppose à l'un des régimes les mieux armés du monde avec "les mains nues", sans recours à la lutte armée, b) «la révolte s'est étendue sans qu'il y ait eu dispersion ou conflit » entre les opposants du régime et $\mathrm{c}$ ) «l'absence d'objectifs à long terme n'est pas un facteur de faiblesse. $\mathrm{Au}$ contraire c'est parce qu'il n'y a pas de programme de gouvernement, c'est parce que les mots d'ordre sont courts qu'il peut $\mathrm{y}$ avoir une volonté claire, obstinée, presque unanime " (FOUCAULT, 2001f, p.703). Comment peut-on parler de volonté collective lorsqu'il s'agit d'un mouvement qui n'a pas de programme ni d'objectif à long terme, encore moins un parti politique, un homme ou une idéologie qui le représente? Dans la réponse à cette question réside tout l'enjeu de la lecture de Foucault. On ne parle de volonté collective que dans la mesure où son effectuation est entièrement anti-politique, c'est-àdire qu'elle échappe à la codifica- tion stratégique et au calcul rationnel de la politique.

Si le philosophe parle de "grève de la politique » à l'égard de ce mouvement c'est parce qu'il y voit une mise en question et en suspens du jeu politique traditionnel. Bien sûr, ceci ne suppose pas qu'il n'y ait pas eu diverses tentatives pour rassurer sur l'avenir politique de l'Iran, comme l'essai d'une " libéralisation accélérée sur place ", visant le retrait provisoire du shah, proposé par les experts américains et les technocrates du régime; ou bien la proposition d'un référendum sur le rejet de la dynastie proposé par les libéraux laïques ; ou, enfin, la même tentative de l'ayatollah Khomeyni d'un référendum qui portait sur l'adoption d'un " gouvernement islamique ». Mais ce qui attire l'attention de Foucault c'est que le caractère non stratégique du mouvement, son côté irréductible à l'exercice du pouvoir politique, a permis aux gens de tous horizons de se rassembler, d'exprimer leur protestation et de devenir autres. C'est pourquoi il affirme: " la question est de savoir quelle forme prend cette volonté nue et massive qui, depuis longtemps, a dit non à son souverain, qui a fini par le désarmer. La question est de savoir quand et comment la volonté de tous va céder la place à la politique, la ques- 
tion est de savoir si elle le veut et si elle le doit " (FOUCAULT, 2001f, p.703-704).

À n'en pas douter, Foucault essaie de saisir ce qui était en train de se passer en Iran, et ses reportages en témoignent. En ce sens, le philosophe ne parle ni du passé ni du futur du soulèvement, mais problématise le complexe panorama iranien et souligne la singularité des événements. Ainsi, lorsqu'il parle de la volonté collective des iraniens, le philosophe se trouve fasciné par le rôle qu'y joue l'ayatollah Khomeyni. Il faudrait préciser, non pas que Foucault voit dans la personne de Khomeyni le leader de la révolution, encore moins qu'il considère que l'ayatollah représente une nouvelle organisation politique entièrement conformée par le clergé ; mais plutôt que le rôle de l'ayatollah a été celui de " chef mythique de la révolte en Iran », c'est-à-dire celui qui a donné à la volonté multiple et collective des iraniens une cible: le départ du shah. D'après Foucault l'ayatollah Khomeyni n'est pas là en Iran puisqu'il vit exilé depuis quinze ans, puis il ne dit rien d'autre que non au régime du shah, enfin il n'est pas un homme politique, il n'a pas l'intention de faire un parti ni beaucoup moins un gouvernement: «Khomeyni est le point de fixation d'une volonté collective »
(FOUCAULT, 2001i, p.716) dans la mesure où cette volonté massive consiste à rejeter le régime du shah et elle se propage à toutes les couches sociales, les mouvements politiques, les religieux, les étudiants, les libéraux, les ouvriers. En fin de compte, il s'agit ici d'une subjectivation collective, d'un mouvement capable de mettre en question la politique ellemême et sa sédimentation dans un régime monarchique. C'est à cet égard que le philosophe pose la question: "quelle place peuton faire, dans les calculs de la politique, à un mouvement comme celui-là? À un mouvement traversé par le souffle d'une religion qui parle moins de l'au-delà que de la transfiguration de ce mondeci » (FOUCAULT, 2001i, p.716).

Le chiisme une expérience spirituelle

Certes, il y a une singularité dans l'islam chiite qui attire l'attention de Foucault, et dans laquelle il trouve un élément mobilisateur pour le soulèvement du peuple iranien. En effet, dans le chiisme le philosophe repère une tradition ésotérique et mystique qui dépasse la pratique orthodoxe d'obéissance à la loi prônée par l'islam exotérique, et qui dans le soulèvement devient toute une force de subjectivation col- 
lective. D'après le principe de l'imâmat, la mort de Mahomet met fin à la lignée des prophètes mais annonce aussi le commencement d'un nouveau cycle de douze imams ou guides spirituels et temporels de la communauté. Exerçant un pouvoir juste et légiférant, les onze premiers Imams sont morts tragiquement, mais le douzième, Mohamad, a disparu et son occultation donne tout le sens à la tradition mystique et ésotérique de la croyance. Les fidèles doivent attendre le retour de l'Imam caché qui fera régner sur la terre l'ordre vrai de l'Islam; mais les croyants, en attendant la parousie de cet Iman, eux, considèrent toute forme de gouvernement comme étant nécessairement imparfaite. C'est ainsi que le philosophe affirme: «le chiisme, en face des pouvoirs établis, arme ses fidèles d'une impatience continue. Il leur souffle une ardeur qui, d'un seul tenant, est politique et religieuse " (FOUCAULT, 2001d, p.686).

S'agissant de la doctrine du chiisme, de son enseignement et de son contenu ésotérique, Foucault y voit une portée assez particulière permettant aux croyants non seulement de se mettre en rapport avec une loi faite par la justice de Dieu, mais aussi d'utiliser les paroles, les exemples et même les martyrs des imams comme une lumière pour délivrer le sens spirituel profond que la loi recèle et qui, pour eux, devient une promesse et garantie pour un changement radical de leurs conditions de vie. D'ailleurs, en ce qui concerne l'organisation du chiisme, il $\mathrm{y}$ a des traits très caractéristiques sous-jacents à la doctrine: l'absence de hiérarchie dans le clergé, l'indépendance des religieux entre eux, mais leur dépendance à l'égard de ceux qui les écoutent, l'importance de l'autorité spirituelle des religieux et leur rôle de guides des croyants.

Évidemment, Foucault est conscient que "le clergé chiite n'est pas une force révolutionnaire [... ] les mollahs ne sont aucunement 'révolutionnaires' " (2001d, p.686) puisqu'ils encadrent la religion officielle depuis le XVIIe siècle et encore, pointe Foucault, le shah s'était entouré d'un groupe de mollahs. Or ceci n'empêche pas que le chiisme, en tant que religion comportant une charge ésotérique et mystique beaucoup plus profonde que son contenu exotérique, ne constitue une force spirituelle produisant des formes de vie, des modes de subjectivation irréductibles à l'exercice stratégique du pouvoir politique:

[la religion chiite] est aujourd'hui ce qu'elle a 
été plusieurs fois dans le passé; la forme que prend la lutte politique dès lors que celle-ci mobilise les couches populaires. Elle fait, de milliers de mécontentements, de haines, de misères, des désespoirs, une force. Elle en fait une force parce qu'elle est une forme d'expression, un mode de relations sociales, une organisation élémentaire souple, et largement acceptée, une manière d'être ensemble, une façon de parler et d'écouter, quelque chose qui permet de se faire entendre des autres et de vouloir avec eux, en même temps qu'eux (FOUCAULT, 2001d, p.688).

\section{Subjectivation collective}

Pourquoi peut-on parler de subjectivation collective lorsqu'il s'agit du soulèvement de sujets qui rendent manifeste leur volonté de rejet du shah en s'appuyant, en partie, sur la fonction de l'islam chiite comme forme de vie? Il est certain que l'on ne peut pas réduire la diversité des acteurs et l'intensité de leurs demandes à un seul acteur; encore moins affirmer que le désir minoritaire de certains groupes se soit vu forcément éclipsé par la volonté majoritaire des religieux. Comme on l'a vu, dans la volonté collective des Iraniens, il faudrait voir l'expression hésitante et disparate d'une société tout en affirmant qu'il y a une volonté collective parfaitement unifiée: " cette volonté a quelque chose de déconcertant. Il s'agit toujours d'une même chose, d'une seule et très précise: le départ du shah. Mais cette chose unique, pour le peuple iranien, cela veut dire tout: la fin de la dépendance, la disparition de la police, la redistribution du revenu pétrolier, la chasse à la corruption, la réactivation de l'islam, un autre mode vie, des nouveaux rapports avec l'Occident, avec les pays arabes, avec l'Asie, etc. " (FOUCAULT, 2001i, p.715). À la limite, on peut dire que la multiplicité des demandes et le fait que celles-ci soient irrécupérables par un parti politique ayant un programme défini, nous suggère qu'il s'agissait là d'une seule demande et d'une seule lutte, à savoir celle d'un changement radical des formes de vie et des conditions d'existence, c'est-à-dire une lutte pour une forme autre de subjectivité.

Dans cette perspective, le rôle de l'islam chiite a été de véhiculer le mécontentement d'une partie de la population, d'être 
la force et la forme d'expression de cette population, voire, dans un moment donné, d'être le lieu de regroupement de l'opposition au régime du shah (FOUCAULT, 2001g). D'ailleurs, on l'a vu, c'est dans le rapport des croyants avec la pratique ésotérique et mystique du chiisme que le philosophe repère une forme de subjectivation assez particulière, celle d'une spiritualité profonde. Cependant, lorsque Foucault parle de subjectivation collective en Iran, il ne se réfère pas qu'aux groupes religieux majoritaires, mais au peuple tout entier qui, en rendant manifeste sa volonté de départ du shah, a dû devenir autre, changer radicalement ses formes de vie, entretenir d'autre type de rapports interpersonnels, générer d'autres types d'identifications avec son histoire, produire d'autres savoirs et d'autres techniques de constitution de soi, en un mot: se réinventer lui-même.

Soulignons qu'il a fallu d'abord un changement dans les formes de subjectivation des iraniens pour que le régime royal du shah tombe. En Iran on assiste ainsi à une certaine inversion du schéma politique/éthique propre aux lectures de Foucault: il n'y a pas eu d'abord un changement dans le diagramme du pouvoir politique et après émergence d'un nouveau type de subjectivité, mais, au contraire, une articulation entre l'éthique, la religion et la spiritualité qui complique et met en question la politique. Si l'on affirme la prééminence de la subjectivation éthique produite en Iran sur le pouvoir politique, c'est parce que l'on considère que l'exigence d'un changement radical de la subjectivité comporte toujours un point de décrochage dans les relations de pouvoir. Ainsi, la lutte des Iraniens pour une nouvelle forme de subjectivité se présente comme incompatible avec les luttes contre l'exploitation économique et contre la domination politique dans la mesure où la subjectivité ouvre elle-même une dimension irréductible aux rapports de force constituant le diagramme de pouvoir.

Foucault précisément considère la subjectivation comme l'aspect non réductible des événements en Iran, et même comme étant leur condition:

En se soulevant, les Iraniens se disaient - et c'est peut-être cela l'âme du soulèvement : il nous faut changer, bien sûr, de régime et nous débarrasser de cet hmme, il nous faut changer ce personnel corrompu, il nous faut changer tout dans le pays, l'organisation politique, le 
système économique, la politique étrangère. Mais surtout, il nous faut changer nous-mêmes. Il faut que notre manière d'être, notre rapport aux autres, aux choses, à l'éternité, à Dieu, etc., soient complètement changés, et il n'y aura de révolution réelle qu'à la condition de ce changement radical dans notre expérience. (FOUCAULT, 2001j, p.748-749).

C'est dans la subjectivité qu'on peut trouver le lieu d'une résistance qui n'est plus antérieure aux relations de pouvoir, mais qui ouvre un écart entre éthique et politique, $\mathrm{y}$ alimente l'antagonisme. En Iran la subjectivité produite dans les événements émerge comme une interruption radicale du jeu politique et de son calcul stratégique, c'est-à-dire comme un mode d'existence spirituelle avec ses propres formes de spatialisation, de temporalisation et de savoirs autres qui sont incompatibles, voire opposés, à ceux de la modernité occidentale. De telle sorte que la subjectivité dont Foucault parle à propos de ces événements ne doit pas être attachée immédiatement au schéma du sujet moderne de la connaissance et du droit; il s'agissait d'une subjectivité collective entièrement fondée une conscience spirituelle et qui, en tant que telle, reste hétérogène au diagramme des relations de pouvoir.

Certes, la singularité des événements en Iran réside dans le caractère subjectif des manifestations, mais en même temps cette singularité comporte un paradoxe : la subjectivation collective qui a fait basculer le régime du shah ne peut être comprise qu'à partir d'une expérience, celle de la spiritualité, laquelle renvoie directement à une conscience religieuse enracinée dans l'orthodoxie millénaire de l'islam chiite. Autrement dit, il semblerait que l'émergence d'une nouvelle forme de subjectivité en Iran ait été rendue possible par une spiritualité et, en tant que telle, cette nouvelle subjectivité serait compatible avec une forme identitaire préalable fixée par les textes sacrés et les lois de l'Islam. Or n'est-ce pas Foucault qui pose la désindividualisation, la désidentification et la dépersonnalisation comme une sorte de condition pour se subjectiver et devenir autre? En réalité, la contradiction n'est qu'apparente. Comme on l'a vu, en effet, le philosophe rappelle la distinction que fait le chiisme entre l'obéissance exotérique à la loi et la vie spirituelle 
profonde. De sorte qu'il y a quelque chose dans l'enseignement du chiisme et dans son contenu ésotérique qui le rend irréductible à une pratique entièrement normée et fixée dans les textes et la loi. Et, en ce sens, l'expérience spirituelle promue par la pratique traditionnelle de la religion ne suppose pas un retour à une forme du passé, mais la promesse et la garantie d'un changement dans les formes de vie des Iraniens face à une modernisation qui se fonde sur un archaïsme:

Quand je dis qu'ils cherchaient à travers l'islam un changement dans leur subjectivité, c'est tout à fait compatible avec le fait que la pratique islamique traditionnelle était déjà là et leur assurait leur identité; dans cette façon qu'ils ont eu de vivre comme force révolutionnaire la religion islamique, il y avait autre chose que la volonté d'obéir plus fidèlement à la loi, il y avait la volonté de renouveler leur existence tout entière en renouant avec une expérience spirituelle qu'ils pensent trouver au cœur même de l'islam chiite (FOUCAULT, 2001j, p.749).
Ainsi, il est fondamental de maintenir cette distinction dans la mesure où elle permet d'entrevoir la dimension où opère la subjectivation en Iran: c'est une chose que la religion entendue comme le rapport externe à la loi, et cela en est une autre que le rapport à soi établi dans la religion. Et précisément c'est dans ce dernier aspect que le philosophe conçoit l'élaboration d'une éthique collective, c'est-à-dire dans la constitution d'un nouveau type de subjectivité et non dans la production d'un sujet assujetti par l'intériorisation d'une norme. De telle sorte, il ne faudrait pas voir la revendication d'une forme passée et religieuse de spiritualité comme constituant une objection majeure en parlant de subjectivation en Iran. S'il y a eu une revendication identitaire, celle-ci jouait plutôt le rôle d'un point d'appui ou de passage, étant un principe de création qui passe par l'affirmation stratégique de l'identité et qui finit forcément par une relation de différenciation et d'innovation avec soi-même et les autres.

Jusqu'ici on a essayé d'esquisser une lecture possible de la façon dont s'est produit une subjectivation collective qui a mis à l'écart la politique traditionnelle iranienne, voire qui a réussi à renverser un régime royal archaïque en produi- 
sant une rupture radicale dans les conditions d'existence matérielle de presque toute une population. Or le mouvement par lequel cette transformation des formes de vie s'est produite est tout à fait subsidiaire d'un autre dont on a parlé précédemment lorsqu'on analysait la notion de "volonté collective " comme étant une grève de la politique. Ainsi et même si l'on veut mettre tout l'accent sur la portée éthique des événements en Iran, on aurait tort de réduire ces événements à l'idée d'une subjectivation collective. Mais alors, de quel type de politique parle Foucault quand il affirme qu'en Iran il y avait une "grève de la politique »? Il s'agit nécessairement d'une politique qui diffère d'une politique comme "calcul stratégique ", mais cette nouvelle politique jouerait-elle un rôle antagoniste vis-à-vis du pouvoir?

\section{III - Spiritualité politique: la spiritualité comme principe de création politique}

Sans doute, parmi les affirmations de Foucault les plus contestées à propos des événements d'Iran, il est celle de "gouvernement islamique " et de "spiritualité politique ». En effet, en discutant avec les religieux, les étudiants, les intellectuels et d'autres acteurs des événements intéressés aux problèmes de l'islam, le philosophe réalise que leur désir n'est ni une libéralisation accélérée sur place ni encore moins l'effectuation d'une "révolution", mais un " gouvernement islamique ». Foucault est tout à fait conscient de l'ambiguïté de l'expression, mais aussi de sa richesse conceptuelle à l'égard des événements qui se déroulaient. Le philosophe avait montré sa fascination par la lecture de Shariatmadari, pour qui le chiisme ne pouvait pas revendiquer l'exclusivité d'un pouvoir temporel, et pour qui le clergé devait garder une position critique à l'égard du pouvoir politique. En ce sens, il considère que par " gouvernement islamique » personne, en Iran, n'entend un régime politique encadré par le clergé tel que cela s'est produit avec l'arrivée des ayatollahs au gouvernement. D'ailleurs, pointe Foucault, par gouvernement islamique certains comprennent un "idéal » et une " utopie »: celles de revenir à ce que l'islam a pu être au temps du prophète, mais aussi d'avancer vers un point lumineux où la fidélité au Coran en tant que force créative se substitue à l'obéissance à la loi. 
Spiritualité politique: noces ou rupture entre spiritualité et politique?

Cependant, Foucault tombe directement sur un autre sens possible de cette expression, à savoir celui d'une "spiritualité politique ». Pas d'abstractions, même pas d'idéaux, le " gouvernement islamique » est une notion très familière aux iraniens dans la mesure où ils en sont acteurs et c'est en son nom qu'ils risquent tout, voire leur vie hors de tout calcul stratégique. Ainsi:

il s'agit d'abord du mouvement qui tend à donner aux structures traditionnelles de la société islamique un rôle permanent dans la vie politique. Le gouvernement islamique, c'est ce qui permettra de maintenir en activité ces milliers de foyers politiques qui se sont allumés dans les mosquées et les communautés religieuses pour résister au régime du shah (FOUCAULT, 2001e, p.692-693).

Philipe Chevalier (2004) signale que la notion de "spiritualité politique » de Foucault peut être entendue comme une sorte de tentative pour nouer ensemble spi- ritualité et politique: il y aurait des noces entre les deux dans la mesure où en Iran, d'après le philosophe, il n'y aurait pas de gouvernement des mollahs - quoique, comme on le sait, la suite des événements lui donnera tort -. Ainsi, Foucault examine comment la spiritualité propre de l'islam chiite en Iran s'est faite de plus en plus politique. La religion en Iran « a été véritablement le vocabulaire, le cérémonial, le drame intemporel à l'intérieur duquel on pouvait loger le drame historique d'un peuple qui met son existence en balance avec celle de son souverain » (FOUCAULT, 2001j, p.746). Ainsi, le chiisme n'oppose pas au régime du shah le " poids de l'inertie » d'une orthodoxie assez restreinte, mais une incroyable capacité pour politiser des structures «indissociablement sociales et religieuses ».

D'ailleurs, c'est dans cette perspective que le philosophe analyse l'absence d'une structure hiérarchique et l'énorme charge ésotérique et mystique dans le chiisme comme étant des traits assez particuliers constituant le gouvernement islamique. Dès les premiers textes, le philosophe se montre captivé par la façon dont les différentes manifestations, célébrations et commémorations religieuses réveillent de plus en plus un ressenti collectif: le départ du 
shah. Dans les mosquées, dans les rues, dans les bazars, avec des haut-parleurs, avec des magnétophones ou avec des cassettes, les mollahs et les fidèles exhortent tout le monde à remettre en question l'état actuel de l'Iran au point que Foucault n'hésite pas à titrer un article «La révolte iranienne se propage sur les rubans des cassettes ». En somme, il y a dans l'agir le plus quotidien des iraniens une capacité à rendre politique des espaces et des expériences inattendues. Par exemple, le philosophe se montre fasciné par l'histoire d'un tremblement de terre qui a fini par détruire complètement une ville, et pour lequel une population a dû se réorganiser sous la conduite d'un religieux, et créer un autre emplacement. Affaire d'organisation? Peut-être, mais d'après Foucault c'est un bon exemple de la capacité de politisation des fidèles, laquelle peut facilement conduire à une innovation politique: "le tremblement de terre avait été une occasion de faire des structures religieuses non pas seulement le point d'encrage d'une résistance, mais le principe de création politique. Et c'est à cela qu'on songe lorsqu'on parle du gouvernement islamique "(FOUCAULT, 2001e, p.693).

Comme volonté politique la notion de gouvernement islamique désignerait un principe de création politique, une "spiritualité politique », c'est-à-dire un mouvement qui rend politique des structures sociales et religieuses de la vie quotidienne des Iraniens. Ici on peut voir une sorte de lien entre la spiritualité de l'islam chiite et la force politique qu'elle entraînait en Iran. Or si la "spiritualité politique " dont parle Foucault ne signifiait pas les noces entre spiritualité et politique, mais une rupture radicale entre le deux comme le suggère Chevalier? En parlant du gouvernement islamique le philosophe décrit un mouvement inverse et réciproque à celui qu'on vient d'esquisser, il s'agirait d'un mouvement:

qui permettrait d'introduire dans la vie politique une dimension spirituelle: faire que cette vie politique ne soit pas, comme toujours, l'obstacle de la spiritualité, mais son réceptacle, son occasion, son ferment. Et c'est là qu'on croise une ombre qui hante toute la vie politique et religieuse d'Iran aujourd'hui (FOUCAULT, 2001j, p.693).

Remarquons la tournure de l'expresion: "spiritualité politi- 
que » et non politique spirituelle. La distinction est fondamentale dans la mesure où elle indique une irruption de la spiritualité dans la politique et, en tant que telle, cette spiritualité ne suppose pas une continuation $\mathrm{du}$ jeu stratégique de la politique, mais sa suspension inattendue. C'est pourquoi le philosophe se demande si cette volonté politique peut rester un principe d'opposition au pouvoir politique, ou si elle doit être comprise comme un principe de réconciliation entre la religion et l'Etat, une contradiction ou le seuil d'une nouveauté. À la limite, la question est d'établir le statut qu'on peut donner à la spiritualité en Iran: "quel sens, pour les hommes qui l'habitent, à rechercher au prix même de leur vie cette chose dont nous avons, nous autres, oublié la possibilité depuis la Renaissance et les grandes crises du christianisme: une spiritualité politique » (FOUCAULT, 2001j, p.693).

\section{Révolution et histoire}

Il s'agit ici d'une spiritualité politique qui a réussi faire tomber le régime du shah et qui en même temps est devenue la force par laquelle s'est produit un changement dans les modes d'existence des Iraniens. Cependant, l'expression est paradoxale dans la mesure où Foucault semble la définir dans une perspective incompatible avec l'usage courant de la politique. C'est une spiritualité qui se dit politique, mais qui s'éloigne radicalement des formes connues de l'organisation politique: elle n'a pas de parti, ni de représentant, encore moins d'idéologie; c'est une spiritualité qui constitue un mouvement " pour se dégager à la fois de la domination par l'extérieur et de la politique à l'intérieur » (FOUCAULT, 2001i, p.716). Une spiritualité qui ne cherche pas à céder la volonté collective qu'elle constitue au politique. À quelle politique se réfère donc cette spiritualité? En fait, si la spiritualité en Iran peut être dite politique, c'est parce qu'elle est d'abord une spiritualité collective, l'affaire de toute une population dans sa disparité.

Mais il ne suffit pas d'affirmer cela. Il faut encore ajouter que cette spiritualité capable de produire une subjectivation collective est politique dans la mesure où la subjectivation collective joue un rôle antagoniste dans le diagramme des relations de pouvoir, c'est-à-dire dans la politique elle-même en tant que rationalité pratique. D'après Foucault l'équivalent de cette spiri- 
tualité dans l'action politique se retrouverait, non pas dans la révolution, mais dans le soulèvement. Il ne s'agit pas seulement là d'une distinction conceptuelle, mais de tout l'enjeu de la lecture par le philosophe des événements en Iran. Certes on reconnait une certaine ambiguité dans l'intitulé de ces événements: "révolution iranienne ", " soulèvement iranien ", " événements en Iran », mais ceci relève de l'usage qu'en fait Foucault et dont il fera une critique ponctuelle dans ses dernières interventions.

"Qu'est pour nous un mouvement révolutionnaire où l'on ne peut pas situer la lutte de classes, où on ne peut pas situer les contradictions internes à la société et où on ne peut pas non plus désigner une avant-garde? » (FOUCAULT, 2001j, p.744). C'est la question que se pose Foucault lorsqu'il entendait dire que l'Iran était le lieu d'une révolution. Le rapport de Foucault avec le marxisme est plutôt critique, notamment à l'égard de l'usage de la méthode dialectique et de la notion de contradiction qu'elle entraînait, et en Iran cette lecture du marxisme ne changera pas. En effet, une partie de l'analyse de l'idée de volonté collective consistait à mettre en évidence l'absence d'un grand affrontement social, d'une tension de lutte violente de classes qui aurait abouti au départ du shah et à la prise du pouvoir politique par les armes. Certes, Foucault n'a pas l'intention de nier la complexité du panorama social iranien et encore moins d'ignorer les possibles contradictions de la société iranienne, mais il remarquait que la manière dont l'événement s'est produit ne témoignait pas de la soi-disant lutte de classes. Même si l'on fait attention au rôle joué par la religion, la lecture de l'événement se complexifie, car la religion n'a pas été une idéologie masquant les contradictions internes de la société iranienne, mais le «drame intemporel » dans lequel s'est déroulé la spécificité de l'événement, c'est-àdire ce que les gens vivent dans une sorte de "théâtre " qu'ils fabriquent au jour le jour et qui constitue leur expérience subjective. L'islam chiite en Iran pendant les événements n'a pas été l'opium du peuple, mais "l'esprit d'un monde sans esprit ».

Si en général une révolution " s'organise selon toute une économie intérieure au temps: des conditions, des promesses, des nécessités ; elle loge donc dans l'histoire, y fait son lit et finalement s'y couche » (FOUCAULT, $2001 \mathrm{~m}$, p.790), alors en Iran il n'y pas eu de révolution. Celle-ci appartient au régime de l'histoire et à l'encadrement causal des faits 
dans une rationalité pratique, laquelle est susceptible d'être analysée dans les termes d'un calcul stratégique. Appeler révolution les événements d'Iran demeure une lecture simpliste qui cache la singularité d'une subjectivation collective qui a fait basculer tout un régime royal et qui, pour un instant, a constitué une volonté politique totalement hétérogène à l'égard du diagramme des relations de pouvoir en Iran. En fin de compte, la révolution relève d'une forme de temporalisation rationnelle et maîtrisable qui requiert en même temps une extension géographique repérable; "le 11 février 1979, la révolution a eu lieu en Iran » (FOUCAULT, 2001k, p.759) écrivait Foucault avec une certaine ironie dans un de ses derniers reportages sur l'événement pour le Corriere della sera en Italie.

Or on aurait tort de réduire la compréhension de la révolution chez Foucault à la critique qu'il fait de l'analyse marxiste de la révolution. Certes, en Iran le philosophe met en question l'existence d'une révolution dans les termes d'une lutte des classes qui, guidée par une avant-garde, aboutirait dans l'instauration d'un gouvernement révolutionnaire. Mais en même temps que le philosophe critique la lecture marxiste, il reconnait l'irréductibilité de la notion de révolution au domaine exclusive de l'analyse socio-politique du XVIIIe et du XIX siècles. En ce sens, la révolution relèverait d'une origine religieuse et spirituelle dont la pensée politique moderne aurait perdu la trace. En Iran, par exemple, la religion n'a pas été le vêtement idéologique de la révolution, mais son expression et sa dramaturgie : elle gère les promesses de l'au-delà, mais aussi la possibilité de transformation de ce monde-ci, elle comporte ses propres formes de temporalisation et spatialisation à partir desquelles les gens vivent et participent avec une certaine conscience dans l'événement de la révolution: "ce sont essentiellement des groupes religieux dissidents qui, à la fin du Moyen Âge et au début de la Renaissance, ont porté cette idée que, à l'intérieur même du monde d'ici-bas, quelque chose comme une Révolution était possible » (SASSINE; FOUCAULT, 2018, p.352).

Soulèvement, spiritualité et interruption de l'histoire

La spiritualité en Iran relevait ainsi d'une autre forme de temporalisation et de spatialisation qui échappaient à la grille d'analyse du marxisme et même aux formes de la connaissance typiquement occidentales. La problématique 
qui se pose en parlant de révolution iranienne explique la difficulté de Foucault à trouver un mot adéquat pour exprimer la singularité politique iranienne: il en parle donc dans les termes d' " insurrection » et de "soulèvement ». "Ce qui échappe à l'histoire, c'est l'instant, la fracture, le déchirement, l'interruption. À la grâce correspond (et répond peut-être), du côté des hommes, le soulèvement » (FOUCAULT, 2001m, p.790) affirmait le philosophe dans un écrit à propos de la mort de M. Clavel, et c'est avec ces mêmes mots qu'il qualifiait le soulèvement iranien: « il faut un arrachement qui interrompt le fil de l'histoire, et ses longues chaînes de raisons, pour qu'un homme puisse, "réellement », préférer le risque de la mort à la certitude d'avoir à obéir » (2001n, p.791).

Loin d'être une action rationnelle dont on peut établir les causes et les effets, le soulèvement est irréductible à une logique stratégique, c'est un événement intempestif dans lequel les hommes se heurtent du pouvoir et, face à lui, opèrent une transformation subjective radicale, au risque de leurs vies. Il y a quelque chose qu'aucune forme de pouvoir ne pourra jamais rendre impossible: le fait même du soulèvement: " la possibilité de ce moment où la vie ne s'échange plus, où les pouvoirs ne peuvent plus rien et où, devant les gibets et les mitrailleuses, les hommes se soulèvent » (FOUCAULT, 2001n, p.791). Ici il n'y a pas de résistances qui opèrent à l'intérieur du diagramme de pouvoir; il ne s'agit pas du schéma pouvoir/résistance du modèle stratégique. L'hétérogénéité du soulèvement réside dans la force imprévisible de son effectuation et dans la capacité de transformation subjective qu'il comporte. Si un homme décide de ne plus obéir à un code ou à une loi au point de préférer la mort, ce n'est pas parce qu'il est complètement piégé par les relations de pouvoir, mais parce que celles-ci avec leurs technologies et dispositifs deviennent inopérantes, en raison de la rupture subjective impliquée dans le choix de l'homme qui se soulève.

En Iran, la spiritualité de l'islam chiite est la force qui donne l'influx au soulèvement et qui constitue l'imaginaire et l'agir de milliers de sujets manifestant dans les rues. À cet égard, l'analyse d'Henri Corbin (1964) sur le chiisme duodécimain et la possible lecture qu'en a fait Foucault sont très significatives pour comprendre la singularité du soulèvement. D'après Corbin la religion islamique ne se limite pas à une interprétation légaliste et judiciaire du texte 
sacré. Cette pratique exotérique est enveloppée d'une pratique beaucoup complexe et vaste, source d'une constante rénovation pour les croyants, à savoir celle de l'ésotérisme. Ainsi, le chiisme se présente essentiellement comme étant l'ésotérisme de l'islam, c'est-à-dire comme une philosophie prophétique constituée sur une eschatologie qui dépasse l'interprétation littérale de la loi et sans laquelle l'islam ne serait qu'une religion positive, opaque et servile. Pour ce faire, l'ésotérisme chiite implique une prophétologie, une imâmologie et une gnoséologie profondément attachées à une réalité spirituelle irréductible à l'interprétation exégétique de la loi.

Il y a des sens cachés ou des sens spirituels provenant des Révélations divines, lesquels ne peuvent être transmis qu'à la façon d'une science qui est, selon Corbin, un " héritage spirituel ». Il en résulte l'existence des degrés de connaissance de l'ordre d'une épiphanie qui constituent une métahistoire - une histoire sans antériorité dans le ciel et qui fait de l'histoire une " hiérohistoire", une histoire sacrée - dans laquelle les croyants réalisent l'attente de la parousie de l'imam caché et qui est subsidiaire d'une eschatologie, d'un savoir sur la fin des temps. En fin de compte l'histoire, pour ceux qui se soulevaient en Iran, ne consistait pas en l'observation, l'enregistrement et la critique de fait empiriques inscrits dans une continuité linéaire maîtrisable, et dont on pourrait établir des causes et des effets. Au contraire l'histoire pour les croyants résultait d'un mode de perception qui dépasse la matérialité des faits empiriques, et d'un mode de perception du suprasensible que conçoit la gnoséologie prophétique, étrangère à l'histoire empirique et à la connaissance scientifique occidentale.

D'après Foucault « les soulèvements appartiennent à l'histoire. Mais, d'une certaine façon, ils lui échappent »(2001n, p.790). Le soulèvement iranien comporte une conscience religieuse inscrite dans une métahistoire, dans une série d'événements messianiques qui place l'événement hors du temps et de l'histoire tels qui ont été conçus par la tradition judéo-chrétienne. Ni les savoirs, ni les techniques ou les pratiques liées au soulèvement ne trouvent leur correspondant respectif dans la philosophie, la théologie et le droit en Occident. À cet égard, il suffit de rappeler la valeur de la vérité dans l'eschatologie chiite: après la prophétie de Mahomet qui clôt le cycle des prophéties établissant les lois, commence un autre cycle de révélation qui 
part des imams, celui de la révélation de la vérité intérieure de la prophétie, grâce auquel les croyants peuvent découvrir le sens spirituel caché dans la doctrine exotérique. Il s'agit ici d'un autre régime de vérité que celui d'occident, c'est une vérité polymorphe au risque de paraître ambigüe, voire contradictoire, dans la mesure où elle ne trouve aucune correspondance dans les principes logiques mis en œuvre pour accéder à la vérité empirique.

Précisément c'est dans ce caractère inachevé de la vérité que Foucault trouve la marque indiscutable du soulèvement iranien et surtout la possibilité permanente d'irruption de la subjectivité dans l'histoire telle que nous la concevons:

A-t-on raison ou non de se révolter? Laissons la question ouverte. On se soulève, c'est un fait; et c'est par là que la subjectivité (pas celle des grands hommes, mais celle de n'importe qui) s'introduit dans l'histoire et lui donne son souffle (FOUCAULT, 2001n, p.793).

Affirmer que la subjectivité frac- ture l'histoire grâce au soulèvement veut dire que cette subjectivité constitue une dimension antagoniste à l'égard du pouvoir politique. En Iran la subjectivation éthique n’a pas été le résultat du diagramme de relations de pouvoir, mais l'émergence d'une nouvelle modalité d'être sujet rendue possible par le chiisme en tant que force spirituelle. En ce sens, si le soulèvement iranien constitue un " déchirement ", une " interruption » dans l'histoire et si l'" âme du soulèvement » consiste en un changement radical de la subjectivité, alors la figure du soulèvement entraîne une nouvelle politique que Foucault repère dans la notion de spiritualité politique. Il s'agirait d'une politique toujours pensée comme une irruption et une déchirure du temps. Une telle politique ne pourrait pas s'installer dans l'histoire, c'est-àdire prendre une forme institutionnelle précise et élaborer un programme d'action, puisqu'elle est fondée sur la spiritualité: elle est toujours référée aux conditions selon lesquelles les individus se transforment et se produisent eux-mêmes pour avoir accès à la vérité. Ainsi cette spiritualité politique reste hétérogène au calcul stratégique d'un pouvoir politique traditionnel. En fin de compte, la spiritualité politique peut être conçue comme étant une 
pratique collective qui exprime des volontés multiples et confuses, c'est-à-dire une politique insaisissable et non identifiable, une politique sans programme ni objectifs à long terme, une politique sans partis, lobbies, idéologies ou représentants.

Certes, l'idée de spiritualité politique et le modèle de soulèvement qu'elle entraîne nous ont permis d'esquisser quelques traits d'une politique autre que celle du pouvoir politique. Une spiritualité politique ainsi conçue comme la possibilité d'affirmation d'une volonté collective vise la transformation des conditions de vie selon un mode que les individus établissent eux-mêmes comme garantie d'une conversion radicale de leur subjectivité, et comme possibilité réelle de création de nouvelles formes de conduites et de rapports aux autres. Cependant, cette radicalité du soulèvement iranien en tant que subjectivation collective, et la force créatrice de la spiritualité comme principe d'innovation politique risquent toujours d'être intégrées aux stratégies globales $\mathrm{du}$ pouvoir politique, comme cela s'est passé dans l'Iran de 1979. Foucault est conscient que la singularité du soulèvement peut être cachée, voire récupérée par des théories globales qui expliqueraient l'événement le rapportant au schéma de la révolution, et c'est dans ce sens qu'il considère que les soulèvements appartiennent à l'histoire:

Vint l'âge de la "révolution ». Depuis deux siècles, celle-ci a surplombé l'histoire, organisé notre perception du temps, polarisé les espoirs. Elle a constitué un gigantesque effort pour acclimater le soulèvement à l'intérieur d'une histoire rationnelle et maîtrisable: elle lui a donné une légitimité, elle a fait le tri de ses bonnes et de ses mauvaises formes, elle a défini les lois de son déroulement; elle lui a fixé des conditions préalables, des objectifs et des manières de s'achever (FOUCAULT, 2001n, p.791).

Après le soulèvement arrive le " grand jour de la révolution " avec son échiquier politique traditionnel: le mouvement par lequel des milliers d'hommes et de femmes se sont heurtés au régime royal du shah en se réclamant d'une spiritualité politique est bientôt annexé par la "stratégie du mouvement religieux», qui aboutit au " gouvernement sanglant d'un clergé intégriste » 
qui commence à retenir, à torturer et à fusiller au nom de l'islam. Il semblerait, remarque Foucault, que le mouvement iranien a subi la « loi »des révolutions: dans tout désir de liberté, il y aurait toujours caché une tyrannie profonde. Si l'histoire n'est constituée que de causes et d'effets et si la révolution n'est qu'une tentative pour " rapatrier " le soulèvement à l'histoire, alors le soulèvement dans l'optique historique ne fait rien d'autre que préparer la terreur de la révolution à venir. Peu importe la violence et l'oppression du nouveau gouvernement si cette terreur se justifie d'emblée dans l'importance du soulèvement qui a précédé l'instauration de ce gouvernement: plus radical est un soulèvement plus catastrophique sera son résultat. C'est dans l'optique de cette critique de la révolution comme intégration stratégique du soulèvement que nous avons débuté cet article. La morale théorique chez Foucault est devenue pour nous une sorte d'impulsion analytique et de grille de lecture pour nous rapprocher du philosophe. Si le stratège, le révolutionnaire par exemple, est l'homme qui, concédant tout l'importance de l'histoire et de ses longues chaînes de raisonnements et de calculs stratégiques, justifie la violence, l'oppression et la mort (peu importe qu'il s'agisse de dix ou de milliers d'hommes) au profit de la volonté générale du peuple, c'est-à-dire de la société dans son sens le plus abstrait, dans ces conditions la morale théorique de Foucault justifie le soulèvement : entièrement tactique il déchire l'histoire et interrompt le flux incessant du temps afin de mettre en évidence notre contingence constitutive et l'infinie capacité de transformation qu'il entraîne.

\section{Éthique et politique antistratégiques}

Après d'avoir établi quelques traits fondamentaux de la lecture de Foucault sur le soulèvement iranien, notamment les catégories de volonté collective et de spiritualité politique, il nous reste plus de questionnements que de certitudes quant à la possibilité de penser une subjectivation éthique collective qui, jouant un antagonisme face au diagramme de relations de pouvoir, fonde une politique autre que celle du pouvoir politique. Certainement, l'analyse de ces deux catégories a été accompagnée d'une brève reconstitution des événements au regard des textes écrits par Foucault, mais on n'a aucunement voulu reconstruire dans sa complexité ni l'évènement en soi ni 
la totalité de l'analyse qu'en a fait le philosophe. On a plutôt privilégié dans un premier temps l'examen de la notion de volonté collective en relation avec la subjectivation collective et ceci dans le cadre d'une expérience qu'avec Foucault on qualifie d'éthique. Il s'agissait en somme de voir la revendication d'un changement dans les formes de vie des Iraniens comme étant l'émergence et la constitution d'une éthique collective conçue à partir d'un rapport à soi non individualisé. Selon nous cette expérience éthique et collective a été capable de produire une conversion, une transformation subjective radicale dans les formes de conduite et de rapport social. Dans un deuxième temps, nous avons voulu montrer comment cette subjectivation éthique collective a pu constituer une politique complètement hétérogène au pouvoir politique traditionnel. À propos de cette nouvelle politique caractérisée à partir de la notion de spiritualité politique et qui se problématise avec l'idée de volonté collective, on a esquissé sa relation avec l'expérience du soulèvement et avec les implications qu'elle entraîne au regard des autres formes de spatialisation, de temporalisation et de savoirs qui placent la subjectivation collective hors de la stratégie politique du diagramme des relations de pouvoir.

Par ailleurs, on peut considérer la subjectivation collective produite en Iran comme une " éthique anti-stratégique »dans la mesure où cette expérience de changement radical dans les formes de vie des Iraniens a toujours comporté un caractère local et contingent. Certes, il y a un rapport à soi qui est objet de modification continuelle, mais il n'est pas considéré comme un point premier et ultime d'autofinalisation. Le rapport à soi et les processus d'individualisation et de personnalisation qu'il entraîne sont conçus comme la forme stratégique que prend une identité déterminée, c'est-à-dire comme des points d'appui ou de passage d'une expérience qui aboutirait forcément à une relation de différenciation et d'innovation avec les autres. En Iran cette relation de différentiation s'est donnée dans les termes d'un rapport à soi collectif, dans lequel le collectif cesse d'être entendu comme une sorte de centre qui lie les sujets dans une hiérarchie, pour devenir un élément de désindividualisation. D'ailleurs, ce rapport à soi collectif s'établi sur la base d'une eschatologie religieuse irréductible au fondement normatif de l'action et à l'obéissance externe à la loi exprimés dans les textes sacrés de l'islam chiite. Bien sûr, dans 
cette expérience éthique, l'action trouve une normativité dans les contenus exotériques de la loi. Cependant, l'interprétation ésotérique de ces contenus exotériques vise à développer une vie spirituelle profonde qui surpasse le fondement normatif de la loi et qui ouvre la possibilité d'une action non réglée et inattendue. Enfin, c'est dans cette perspective qu'on doit concevoir le rôle de la vérité comme l'élément transversal et différenciateur de cette éthique antistratégique. La vérité pour laquelle les Iraniens se sont heurtés au pouvoir politique en risquant leurs vies, et grâce à laquelle il est possible de parler de subjectivation collective, est une vérité provenant des prophéties et d'une métahistoire sacrée, qui ne trouve aucune correspondance définitive avec les régimes de vérité en Occident. Ainsi, cette vérité autre des iraniens comporte des pratiques et des discours qui, grâce à leur polyvalence tactique, n'arrivent jamais à constituer des grandes lignes de force dans une " configuration stratégique ».

Une telle éthique antistratégique joue un rôle, non de coimplication, mais d'antagonisme à l'égard du pouvoir politique $\mathrm{du}$ gouvernement des autres. Face à cette politique stratégique, la subjectivation éthique collective se constitue comme le point d'émergence d'une politique autre, d'une spiritualité politique. Celle-ci consisterait en une pratique collective qui exprime des volontés multiples et confuses, une politique non identifiable, sans programme ni objectifs à long terme, une politique sans partis ni représentants. En somme, une politique antistratégique serait une nouvelle politique chargée de transgresser les régimes de production de la vérité, en détachant le pouvoir de la vérité des formes d'hégémonie propres qu'on construit dans n'importe quel type de conformation sociale.

\section{Referências}

BENSAÏD, D. "Grandeurs et misères de Deleuze et Foucault". Le site Daniel Bensaïd. (Blogue d'internet), entre 2007 et 2009, disponible sur [http: / / danielbensaid.org/Im-politiques-de-Foucault]: visité le [14 novembre 2014].

CORBIN, H. "Le shiisme et la philosophie prophétique". In: Histoire de la philosophie islamique. Paris: Gallimard, 1964, p. 41-109. 
CHEVALIER, PH. "La spiritualité politique, Michel Foucault et l'Iran". Ceras, Revue, projet $\mathrm{n}^{\circ} 281$, juillet, 2004.

FOUCAULT, M. Histoire de la sexualité I: L'usage des plaisirs. Paris: Gallimard, 1984.

FOUCAULT, M. Histoire de la sexualité II: Le souci de soi. Paris: Gallimard, 1984.

FOUCAULT, M. Dits et écrits II 1976-1988. Paris: Gallimard, 2001.

FOUCAULT, M. "Préface". In: Dits et écrits II. Op.cit. n.189, p.133-136, 2001 a.

FOUCAULT, M. "L'armée, quand la terre tremble". In: Dits et écrits II. Op.cit. n.241, p.662-669, 2001b.

FOUCAULT, M. "Le chah a cent ans de retard". In: Dits et écrits II. Op.cit. n.243, p.679-683, 2001c.

FOUCAULT, M. "Téhéran: la foi contre le chah". In: Dits et écrits II. Op.cit. n.244, p.683-688, 2001d.

FOUCAULT, M. "A quoi rêvent les iraniens? ”. In: Dits et écrits II. Op.cit. n.245, p.688-694, 2001e.

FOUCAULT, M. "Un révolté à mains nues". In: Dits et écrits II. Op.cit. n.248, p.701-704, 2001f.

FOUCAULT, M. "Défi à l'opposition". In: Dits et écrits II. Op.cit. n.249, p.704-706, 2001g.

FOUCAULT, M. "Les reportages d'idées". In: Dits et écrits II. Op.cit. n.250, p.706-707, 2001h.

FOUCAULT, M. "Le chef mythique de l'Iran". In: Dits et écrits II. Op.cit. n.253, p.713-716, 2001 i.

FOUCAULT, M. "L'esprit d'un monde sans esprit". In: Dits et écrits II. Op.cit. n.259, p.743-755, 2001j.

FOUCAULT, M. "Un poudrière appelée islam". In: Dits et écrits II. Op.cit. n.261, p.759-761, 2001k.

FOUCAULT, M. "Vivre autrement le temps". In: Dits et écrits II. Op.cit. n.268, p.789-790, 2001m.

FOUCAULT, M. "Inutile de se soulever?". In: Dits et écrits II. Op.cit., n.269, p.790-798, 2001n.

FOUCAULT, M. Sécurité, territoire, population. Cours au Collège de France (1977-1978). Paris: Gallimard-Seuil, 2004.

RAMBEAU, F. “La política espiritual”, Ciencia Política, Bogotá, v.10, n. 19, p. 197-192, janvier-juin 2018.

SASSINE, F.; FOUCAULT, M. "Entretien inédit avec Michel Foucault 1979”. Foucault Studies. n.25, pp. 351-378, octobre 2018. 
Recebido: 18/02/2019

Aprovado: 19/04/2019

Publicado: 13/05/2019 